\section{Safety of drugs used in the treatment of osteoporosis}

\author{
Cora McGreevy and David Williams
}

Abstract: A number of drug classes are licensed for the treatment of osteoporosis including bisphosphonates, recombinant human parathyroid hormone (PTH), strontium, hormone replacement therapy (HRT), selective oestrogen receptor modulators (SERMS) and denosumab. This review discusses the safety of osteoporosis treatments and their efficacies. Recent concerns about the safety of calcium and high-dose vitamin D are discussed. Bisphosphonates have substantial postmarketing experience and a clearer picture of safety issues is emerging. Along with the well recognized effects on the gastrointestinal tract and kidney function, recently described adverse effects such as osteonecrosis of the jaw, oesophageal cancer, atrial fibrillation, subtrochanteric femur fractures and ocular complications of bisphosphonate therapy are discussed. Therapy with PTH is limited to two years' duration because of the development of osteogenic sarcomas in animal studies, which appeared related to dose, duration and timing of therapy. Strontium should be used with caution in patients with renal impairment and its use has been associated with venous thromboembolism. The role of HRT and SERMs in the treatment of postmenopausal osteoporosis is restricted as a result of an increased risk of stroke, venous thromboembolism and breast cancer. Postmarketing experience with denusomab is limited but a number of potential safety concerns including osteonecrosis of the jaw are emerging. All of these drugs have been proven to reduce fractures. The decision to use a drug to reduce fracture risk should be based on risk-benefit analysis of the drug and its suitability for individual patients.

Keywords: adverse effects, bisphosphonates, drug safety, osteoporosis, recombinant human parathyroid hormone, strontium, vitamin $D$

\section{Introduction}

Osteoporosis is typified by low bone mass and changes in the architecture of bone which results in increasing bone fragility and fractures [Ahmed and Elmantaser, 2009]. It is the most common bone disease in the Western world. Bone mineral density (BMD) increases in both men and women from early childhood and peaks in early adulthood. With ageing, there is a gradual loss of bone, with an accelerated loss apparent around the perimenopausal period in women. The disease is asymptomatic until a fracture is sustained, which frequently results in pain and disability. Optimization of bone density by means of risk factor modification, peak bone mass attainment, use of antiosteoporotic therapies and falls prevention strategies are key to osteoporosis prevention. In health economic terms, the disease burden is considerable and growing with the ageing population. By 2020, the yearly cost of osteoporotic fractures is estimated to grow by $20 \%$ to over $£ 2.1$ billion in the UK [Burge et al. 2001].

\section{Pathophysiology}

The continual remodelling of bone architecture is the basis of the development of osteoporosis [Seeman and Delmas, 2006]. The interactions between the two main cell types, osteoblasts and osteoclasts, which are both derived from the haematopoietic system determines the balance between bone resorption and bone deposition. Osteoclasts function by acidifying their domain by means of proton secretion into the extracellular space with a resulting disintegration of bone mineral [Blair et al. 1989]. Osteoblasts lay down new bone mineral through a process that has not yet been fully elucidated. The balance between the activities of osteoblasts and
Ther Adv Drug Saf (2011) 2(4) 159-172 DOI: $10.1177 /$ 2042098611411012

(C) The Author(s), 2011. Reprints and permissions: http://www.sagepub.co.uk/ journalsPermissions.nav
Correspondence to: Dr Cora McGreevy Department of Medicine for the Elderly, RCSI and Beaumont Hospital, Dublin 9, Ireland coramcgreevyarcsi.ie

Professor David Williams Department of Medicine for the Elderly, RCSI and Beaumont Hospital, Dublin, Ireland 
osteoclasts regulates bone production, maintenance and destruction.

A number of hormones including oestrogen and parathyroid hormone (PTH) are involved in the process of bone formation. Oestrogen is believed to play a major role in the actions of osteoblasts and osteoclasts via receptors located on the surface of these bone cells [Zallone, 2006]. When oestrogen levels decrease, osteoclasts develop a more corrosive action which can lead to the loss of large cancellous sections of bone [Eriksen et al. 1999]. This mechanism has been attributed to the loss of the proapoptotic effect of oestrogens on osteoclasts [Hughes et al. 1996]. The overall effect of PTH is to raise plasma levels of calcium, through bone resorption. This is achieved by PTH-mediated differentiation of preosteoblast cells to the osteoblast form. The osteoblasts generate interleukin-6 (IL-6), which in turn stimulates preosteoclasts to differentiate into osteoclasts [Kroll, 2000].

The receptor activator of nuclear factor $\kappa B$ (RANK) cell surface receptor provokes osteoclast activity by causing osteoclast precursor cells to mature into fully differentiated osteoclasts when RANK comes in contact with its corresponding RANK ligand (RANKL) [Yasuda et al. 1998]. This ligand is manufactured by osteoblasts and is one of a number of signalling molecules that promotes communication between different bone cell types, thereby contributing to the process of bone remodelling. RANKL inhibitors have recently been registered for the treatment of osteoporosis in humans and will be discussed later in this article.

\section{Treatment of osteoporosis}

National Osteoporosis Foundation (NOF) guidelines recommend that pharmacological therapy should be limited to postmenopausal women and men aged 50 years or older who present with the following [NOF, 2010]:

1. A hip or vertebral fracture (the vertebral fracture may be clinical or morphometric, i.e. identified on a radiograph alone).

2. T-score less than -2.5 at the femoral neck, total hip, or spine after appropriate evaluation to exclude secondary causes.

3. Low bone mass ( $T$-score between -1.0 and -2.5 at the femoral neck, total hip, or spine) and: a. a 10-year probability of hip fracture of $3 \%$ or more or

b. a 10-year probability of any major osteoporosis-related fracture of $20 \%$ or more based on the UK-adapted World Health Organization (WHO) algorithm [WHO, 2006].

These guidelines incorporate the knowledge that individuals who have already suffered a fragility fracture are at highest risk of future fractures, and the guidelines recommend further evaluation and treatment for this cohort. Fragility fracture is a type of fracture that occurs as a result of a low trauma event, such as a fall from standing height or less. There are three characteristic fragility fracture sites: vertebral fractures, neck of the femur, and Colles fractures of the wrist [Klotzbeucher et al. 2000]. Individuals who have sustained previous fragility fractures are at twice the risk for a subsequent fracture, irrespective of the previous fracture site [Klotzbeucher et al. 2000].

\section{Vitamin D and calcium supplements: efficacy}

Many studies have linked vitamin D with bone health, muscle function and postural stability [DIPART Group, 2010; Bischoff-Ferrari et al. 2009, 2006; Tang et al. 2007]. A meta-analysis of 12 randomized controlled trials (RCTs) of nonvertebral fractures $(n=42,279)$ and eight randomized controlled trials of hip fractures $(n=40,886)$ compared oral vitamin $\mathrm{D}$ (with or without calcium) with either calcium alone or placebo. The authors concluded that prevention of nonvertebral fractures with vitamin $\mathrm{D}$ is dose dependent, and a dose of greater than $400 \mathrm{IU} /$ day reduced fractures by at least $20 \%$ in individuals aged 65 years or older [Bischoff-Ferrari et al. 2009].

Coprescription of calcium and vitamin D is now recommended for the treatment of osteoporosis [Tang et al. 2007], and current guidelines advocate that older adults receive at least $800-1000$ IU per day of vitamin D along with calcium to reduce risk of falls and fractures [Dawson-Hughes et al. 2010]. Current NOF guidelines recommend that premenopausal women and men aged 50 years and younger without risk factors for osteoporosis should receive a total of $1000 \mathrm{mg}$ of elemental calcium daily [NOF, 2010]. Postmenopausal women, men older than 50 years, and individuals with risk factors for osteoporosis should receive a total of 
$1200-1500 \mathrm{mg}$ of calcium daily. Calcium consumed from dietary intake is deemed to be the optimal source but calcium supplements are recommended if dietary sources are inadequate.

National Institute for Health and Clinical Excellence (NICE) guidelines advocate that sufficient levels of calcium and vitamin $\mathrm{D}$ are needed to ensure maximum effects of the pharmacological treatments for osteoporosis. The guidelines state that calcium and/or vitamin D supplementation should be prescribed unless clinicians are 'confident that patients receiving osteoporosis treatment have an adequate calcium intake and are vitamin D replete' [NICE, 2008].

\section{Calcium and vitamin D supplements: safety issues}

When prescribed alone, calcium supplements have been shown to increase BMD but have limited effect on fracture reduction and have been associated with a possible increase in cardiovascular events [Bolland et al. 2010]. An increased risk of myocardial infarction was noted in those on calcium supplements alone after a median follow up of 3.6 years. Calcium supplements have been shown to improve blood pressure and cholesterol levels [Reid et al. 2010]. The relevance of any potential detrimental effect of calcium alone on cardiovascular disease is uncertain given the recommendation that calcium alone is no longer advised as an appropriate treatment for osteoporosis [Dawson-Hughes, 2010]. A related editorial recommends that individuals with osteoporosis should not be prescribed calcium supplements, either alone or combined with vitamin $\mathrm{D}$, unless they are on concomitant therapy for osteoporosis [Cleland et al. 2010]. In the light of the findings by Bolland and colleagues, the authors always coprescribe calcium with vitamin $\mathrm{D}$ and await further research in this area with interest.

A recently published RCT found that older women who received a single annual dose of 500000 IU of cholecalciferol versus placebo experienced $15 \%$ more falls and $26 \%$ more fractures than their counterparts in the placebo group [Sanders et al. 2010]. This study used the largest single dose of vitamin D (500 $000 \mathrm{IU})$ reported in any large RCT, which suggests that the increase in adverse events may be dose related. However, a double-blind RCT of 100000 IU oral vitamin D supplementation versus placebo every 4 months over 5 years found that total fracture rate was reduced by one fifth and fractures in major osteoporotic sites were reduced by one third [Trivedi et al. 2003].

Given the improved outcomes in the 4-monthly regimen versus the annual dosing schedule, the authors feel that a large annual dose is best avoided and that divided doses throughout the year may be the optimal treatment.

\section{Bisphosphonates: efficacy}

Bisphosphonates are synthetic analogues of pyrophosphate with high affinity for hydroxyapatite crystals [Keen, 2007]. By binding at sites of active remodelling, they inhibit osteoclastic resorption, thereby reducing bone resorption. Absorption of oral bisphosphonates is less than $5 \%$, with subsequent bone uptake varying from $20 \%$ to $80 \%$, with the remainder being rapidly excreted through the kidney [Keen, 2007]. They have a short plasma half life but are very slowly eliminated from bone and have a half life of several years. Bisphosphonates have become established as the first-line treatment for osteoporosis.

\section{Alendronate}

Alendronate is given at doses of either $10 \mathrm{mg} /$ daily or $70 \mathrm{mg} /$ weekly and is available in combination with $5600 \mathrm{IU}$ of vitamin $\mathrm{D}$.

The Fracture Intervention Trial (FIT) included over 2000 women with an existing vertebral fracture and over 4400 women with osteoporosis without a vertebral fracture [Black et al. 1996]. Results showed that amongst those with existing vertebral fractures, $8 \%$ in the alendronate group had $\geq 1$ new vertebral fractures at 3 years versus $15 \%$ in the placebo group. In the FIT2 substudy, women without vertebral fractures at baseline but with a femoral neck BMD T-score less than -1.6 were randomized to receive alendronate or placebo for 4 years. Women in the alendronate group were found to have a decreased risk of subsequent vertebral fractures of $44 \%$ [Cummings et al. 1998].

The FOSamax Interventional Trial (FOSIT) focused on the role of alendronate in reducing the incidence of nonvertebral fractures and found that individuals in the alendronate group were found to have greater gains in BMD in both the lumbar spine $(4.9 \%)$ and the femoral neck $(2.4 \%)$ at 12 months in comparison to placebo [Pols et al. 1999]. Furthermore, the rate of 
nonvertebral fractures was significantly lower in the alendronate group (19 versus 37 with fractures).

\section{Risedronate}

Risedronate is given in doses of either $5 \mathrm{mg} /$ daily or $35 \mathrm{mg} /$ weekly. It has similar indications and contraindications to alendronate. A meta-analysis of eight RCTs which examined the efficacy of risedronate in the treatment of postmenopausal osteoporosis found that risedronate significantly lowered the risk for both vertebral and nonvertebral fractures [Cranney et al. 2002].

Alendronate and risedronate were directly compared in the Fosamax Actonel Comparison Trial (FACT), which looked at BMD gains and suppression of bone turnover markers in over 1000 women over 1 year [Rosen et al. 2005]. The results showed that alendronate was superior in the area of BMD gains at all sites (lumbar spine, femoral neck and total hip) and was associated with greater reductions in bone turnover markers than risedronate.

\section{Ibandronate}

Ibandronate is given as either $150 \mathrm{mg}$ monthly or $3 \mathrm{mg}$ intravenously every 3 months.

In the Ibandronate Osteoporosis vertebral fracture trial in North America and Europe (BONE) study, patients were randomized to receive either oral ibandronate or placebo in daily doses of $2.5 \mathrm{mg}$ or intermittent doses ( $20 \mathrm{mg}$ alternate days for 12 doses), 3 monthly. Results showed that after a 3-year period, individuals receiving daily or intermittent ibandronate had greater gains in BMD than those in the placebo group [Chesnut et al. 2004]. Both regimes of oral ibandronate resulted in a statistically significant reduction in the risk of vertebral fractures and showed a diminution in bone turnover markers with a reduction of $50-60 \%$ seen at 3 months [Delmas et al. 2004].

\section{Zoledronic acid}

This yearly intravenous infusion is licensed for osteoporosis in postmenopausal women. Zoledronic acid is well tolerated with the most frequent side effects being an acute flu-like illness in the first 3 days which can include joint and muscle pain.

One RCT which compared different doses of zoledronic acid with placebo for the treatment of postmenopausal osteoporosis found that there were significant improvements in BMD at both the lumbar spine and femoral neck in all zoledronic acid dosing regimens compared with placebo [Reid et al. 2002]. In a more recent study, zoledronic acid decreased the risk of vertebral fracture by $70 \%$ over a 3-year timeframe versus the placebo group [Black et al. 2007].

\section{Bisphosphonates: safety issues}

\section{Oral bisphosphonates and oesophagitis}

Following ingestion of oral bisphosphonates it is recommended that patients sit upright for $30 \mathrm{~min}$ because of the recognized risk of oesophagitis. Oral bisphosphonates are contraindicated in patients with oesophageal abnormalities and diseases that slow gastric emptying.

Caution is advised with patients with renal insufficiency [glomerular filtration rate $(\mathrm{GFR})<35 \mathrm{ml} / \mathrm{min}]$.

Oral bisphosphonates and oesophageal cancer Gastrointestinal (GI) effects of oral bisphosphonates are well recognized and vary from mild nausea and heartburn to erosive oesophagitis and ulceration. Recent evidence has suggested a possible increase in the incidence of oesophageal cancer with use of oral bisphosphonates [Wysowski, 2009]. In a recent study, in which data were accessed via the UK General Practice Research Database, the rates of oesophageal and gastric cancer were compared in 40,000 patients treated with oral bisphosphonates over a 10 -year period with the rate of GI malignancy in a control group [Cardwell et al. 2010]. No significant association between oral bisphosphonate use and risk of oesophageal or gastric cancer was found. However, a recent case control analysis which also used data from the UK General Practice Research Database found patients who had received 10 or more prescriptions for oral bisphosphonates were at increased risk for oesophageal cancer and a similar increased risk was noted in those who had received prescriptions over a 5-year period [Green et al. 2010]. In the Western world, the incidence of oesophageal cancer at age 60-79 is approximately 1 per 1000 population over 5 years, and the authors approximate that this increases to about 2 per 1000 with 5 years' intake of oral bisphosphonates. The observational study period is almost double in the analysis by Green and colleagues compared with the study by Cardwell and colleagues (on average 7.7 
versus 4.5 years). Limitations of both studies were that diagnoses were not confirmed by medical records and no information on compliance or adherence was established. The UK Commission on Medicines recommended that no definite causal association between oral bisphosphonates and oesophageal cancer could be ascertained from this study. More studies are needed in particular to examine the potential links between use of different types of oral bisphosphonates and the risk of oesophageal malignancy.

Given the well documented adverse GI side effects of oral bisphosphonates, clinicians should routinely ask patients about GI disorders prior to prescribing, and emphasize the importance of adherence to instructions on ingesting these products [Wysowski, 2010]. Bisphosphonates have low oral bioavailability and food impairs their absorption. Calcium supplements and antacids also decrease their absorption and should be taken at least $30 \mathrm{~min}$ after bisphosphonates.

\section{Bisphosphonates and renal impairment}

Bisphosphonates are predominantly renally excreted, giving concern about the potential for accumulation of these agents in patients with chronic kidney disease (CKD). In the early bisphosphonate studies, patients with CKD were excluded on the basis of their serum creatinine levels. In more recent trials, creatinine clearance is calculated as part of exclusion criteria [Miller et al. 2005].

Prolonged treatment with alendronate has been associated with adynamic bone disease and atypical long bone fractures in postmenopausal osteoporosis [Odvina et al. 2005]. This has given rise to concern that further bone turnover reduction by means of bisphosphonate treatment might lead to a higher risk of fracture, given the already high prevalence of adynamic bone disease in the renally impaired population [Cunningham et al. 2004]. Further analysis of the FIT trial data showed that patients with CKD who had received alendronate had significant improvement in BMD along with a decreased fracture rate comparable to that seen in patients with a higher creatinine clearance [Jamal et al. 2007]. Analysis of trials involving risedronate showed that almost $45 \%$ had a GFR of $50-80 \mathrm{ml} / \mathrm{min}, 41 \%$ had a GFR of $30-50 \mathrm{ml} / \mathrm{min}$ and $6 \%$ had a GFR less than $\mathrm{ml} / \mathrm{min}$. Individuals with lower creatinine clearance $(<30 \mathrm{ml} / \mathrm{min})$ who were treated with placebo had a higher incidence of new fractures in contrast with those with moderate (creatinine clearance $>30$ to $<50 \mathrm{ml} / \mathrm{min}$ ) and mild ( $>50$ to $<80 \mathrm{ml} / \mathrm{min}$ ) renal impairment [Andersen and Kukreja, 2008]. Patients with severe renal impairment who were treated with $5 \mathrm{mg}$ daily risedronate were found to have a greater reduction of fracture risk compared with those with mild and moderate renal impairment [Miller et al. 2005].

Renal safety when using intravenous bisphosphonate in kidney disease has been looked at in a number of studies. Bisphosphonate nephrotoxicity is both dose and infusion time dependent, and its occurrence can be limited by increasing the time interval between doses [Perazella and Markowitz, 2008]. A toxic form of acute tubular necrosis has been reported with the use of zoledronic acid infusions in patients with malignancy [Markovitz et al. 2003]. Postmenopausal osteoporotic women who received yearly infusions of zoledronic acid over 3 years ( $5 \mathrm{mg}$ given intravenously over $15 \mathrm{~min}$ ) showed transient increases in serum creatinine but no significant difference in creatinine clearances noted over the study period [Boonen et al. 2008]. Strict adherence to guidelines for monitoring serum creatinine prior to each treatment, withdrawing therapy in the setting of renal insufficiency, and reducing doses in patients with preexisting CKD are crucial in avoiding severe nephrotoxicity.

From retrospective analysis, bisphosphonates appear to be relatively safe and efficacious when standard doses are used to treat osteoporotic patients with stages 2 and 3 CKD. US Food and Drug Administration (FDA) approval has been issued to sanction the use of bisphosphonates for osteoporosis prevention and treatment in patients with a creatinine clearance of greater than $30-35 \mathrm{ml} / \mathrm{min}$. However, efficacy and safety of this class of drugs has not yet been examined in patients with stage $5 \mathrm{CKD}$ [Andersen and Kukreja, 2008].

\section{Oral bisphosphonates and atypical subtrochanteric femur fractures}

A number of recent reports have linked prolonged use of alendronate with an increased risk of atypical stress fractures [Lenart et al. 2008]. Causality is less well established between atypical fractures and other bisphosphonates, which may be primarily due to the lack of availability of longterm safety data in bisphosphonates other than 
alendronate. The incidence of these insufficiency type fragility fractures is low and they tend to present with certain characteristics, such as minor or no trauma and prodromal thigh pain [Abrahamsen et al. 2009]. They tend to occur after prolonged bisphosphonate therapy with median duration of 3-8 years. Other characteristics included delayed healing and bilateral presentation. Retrospective data analysis from two observational studies of osteoporotic patients revealed that subtrochanteric femur fractures shared many characteristics with typical osteoporotic hip fractures, including patient age, sex, and trauma pattern [Abrahamsen et al. 2009]. In the event of a stress fracture, current recommendations include the immediate discontinuation of bisphosphonate therapy and imaging of the contralateral femur [MHRA, 2009].

Many clinicians now advocate a drug holiday after 5-10 years of bisphosphonate therapy [Watts and Diab, 2010]. Research suggests that bisphosphonates provide some residual antifracture effect after being stopped and this effect appears to be maintained for 2-5 years after stopping the drug [Schmidt et al. 2010]. More research is necessary to establish the significance of supersuppression of bone turnover by bisphosphonate therapy [Chaiamnuay and Saag, 2006].

With recent evidence from published literature linking long-term bisphosphonate therapy with femoral stress fractures, the American Society for Bone and Mineral Research (ASBMR) have appointed a task force to focus on issues related to this problem [Shane et al. 2010]. This international, multidisciplinary expert group has introduced strict criteria relating to major and minor features of complete and incomplete atypical stress fractures. The taskforce recommends that the following major features must be present to define a femoral fracture as atypical: 'location in the subtrochanteric region and femoral shaft, transverse or short oblique orientation, minimal or no associated trauma, a medial spike when the fracture is complete, and absence of comminution'. Minor features including 'cortical thickening, a periosteal reaction of the lateral cortex, prodromal pain, bilaterality, delayed healing, comorbid conditions, and concomitant drug exposures, including bisphosphonates, other antiresorptive agents, glucocorticoids, and proton pump inhibitors' should also be sought to confirm the diagnosis of atypical femoral fractures [Shane et al. 2010]. Furthermore, the task force advocates that specific diagnostic and procedural criteria be established and that an international registry be created to allow more detailed research into the predisposing risk factors and optimal management of these atypical fractures.

\section{Intravenous bisphosphonates}

and hypocalcaemia

A number of case reports have been published detailing the development of hypocalcaemia after receiving intravenous bisphosphonates [Peter et al. 2004]. These cases have primarily involved patients with hypercalcaemia of malignancy. The mechanism for the hypocalcaemia appears to be a bisphosphonate induced blunting of the normal response of the parathyroid gland to low serum calcium levels and the hypocalcaemia is usually transient. Two of the four cases reported by Peter and colleagues also had vitamin $\mathrm{D}$ deficiency which further contributed to their hypocalcaemia.

Bisphosphonates and osteonecrosis of the jaw Osteonecrosis of the jaw (ONJ) is a severe and disabling bone disease of the jaw and includes the maxilla and mandible. ONJ related to bisphosphonates is characterized by exposed or dead bone in the jaw for more than 8 weeks' duration, in a patient who has been or is currently on bisphosphonate therapy and has not had radiotherapy on the jaw. It is a form of avascular necrosis whereby the blood supply to localized areas of the jaw is reduced. It is mainly noted in patients who have received high doses of bisphosphonates, particularly in the setting of dental procedures post chemotherapy. A recent review summarized 368 cases of ONJ with the vast majority of reported cases occurring in patients with malignant bone disease [Woos et al. 2006]. Of the three cases of ONJ that occurred after treatment with bisphosphonates for Paget's disease, two had been prescribed inappropriately high doses of bisphosphonates [Bolland et al. 2006]. Of a total of 63 cases of ONJ patients treated with bisphosphonates reported in 2004, seven patients had taken bisphosphonates for osteoporosis without a history of malignant disease. The European Medicines Agency reviewed the data on bisphosphonate-related ONJ in 2009 and concluded that individuals receiving intravenous bisphosphonates for malignancy were at higher risk for developing the condition compared with those taking oral bisphosphonates for osteoporosis or Paget's disease 
[CHMP, 2009]. In the HORIZON-PFT trial, only one case of ONJ was reported.

The majority of cases of bisphosphonate-associated ONJ occurred after dental procedures or surgery and the remaining cases occurred spontaneously [Rustemeyer and Bremerich, 2010]. It has been suggested that ONJ following bisphosphonate therapy may be linked to a defect in wound healing caused by inhibition of bone turnover induced by osteoclast inhibition. Because bisphosphonates are selectively deposited in areas of high bone turnover, it is possible that this complication is limited to the jaw bones as there have been no published cases outside the craniofacial skeleton. Many of the reported cases involved patients with false dentition which may be a source of local trauma. Aggressive treatment of periodontal infections and good oral hygiene are therefore crucial and recommended prior to commencing bisphosphonate therapy to help prevent ONJ.

On commencing bisphosphonate treatments, the authors advise patients on the importance of good oral hygiene, regular dental examinations and to report any oral symptoms such as dental pain or inflammation.

\section{Bisphosphonates and atrial fibrillation}

Atrial fibrillation (AF) came to light as a potential adverse effect of bisphosphonate therapy in the HORIZON Pivotal Fracture Trial in which there was a higher risk of $\mathrm{AF}$ in participants taking zoledronic acid than in recipients of placebo [Black et al. 2007]. Most AF events were reported more than 1 month after the initial infusion, by which time zoledronic acid would be at extremely low concentrations in the circulation. Fifty patients in the zoledronic acid group developed $\mathrm{AF}(1.3 \%)$, in comparison with 20 patients $(0.5 \%)$ in the placebo group $(\mathrm{p}<0.001)$, whereas the incidence of any AF events was similar between treatment and placebo groups $(2.4 \%$ versus $1.9 \% ; \mathrm{p}=0.12)$. In the Recurrent Fracture arm of the HORIZON trial there was no evidence of this potential adverse event because the frequency of $\mathrm{AF}$ was similar in both the zoledronic acid and placebo groups $(1.1 \%$ versus $1.3 \% ; \mathrm{p}=0.84)$ [Lyles et al. 2007]. In 2008, the FDA advised that a review of the clinical evidence showed no clear association between bisphosphonate treatment and $\mathrm{AF}$ and the FDA's MedWatch advocated that clinicians should not alter their prescribing practices for bisphosphonates [FDA, 2008].

\section{Bisphosphonates and ocular complications}

Previous studies have shown that pamidronate disodium can cause uveitis, nonspecific conjunctivitis, episcleritis, or scleritis [Macarol and Fraunfelder, 1994]. Further analysis of the published literature on other bisphosphonates available indicates that in rare instances, bisphosphonates therapy can lead to serious ocular adverse reactions. The variance in the number of ocular side effects attributed to each bisphosphonate may be an indication of the popularity of each medication [Fraunfelder et al. 2003]. Of note, no case of unilateral or bilateral scleritis that developed in a patient on bisphosphonate therapy resolved, regardless of therapy, until the offending medication was stopped. Current clinical guidelines regarding bisphosphonates advocate rapid ophthalmological referral if individuals present with eye pain or loss of vision. Nonspecific conjunctivitis rarely necessitates pharmacological intervention and its intensity on next exposure to a bisphosphonate. Bisphosphonate therapy must always be discontinued in order for scleritis to fully resolve [Fraunfelder and Fraunfelder, 2003].

\section{Recombinant human parathyroid hormone}

PTH is responsible for stimulating bone turnover and can induce renewed modelling and increase cortical thickness of bone. Recombinant human PTH is licensed as a treatment for postmenopausal osteoporosis.

\section{Teriparatide}

Teriparatide is an anabolic product that contains human PTH. Teriparatide is recommended for the treatment of osteoporosis in patients with an increased risk of fracture. The licensed dose is $20 \mu \mathrm{g}$ /day with maximal duration of 24 months. One study randomized 2532 postmenopausal women (mean age 64 years) with osteoporosis to receive either PTH or placebo. The results showed that women in the PTH group had a decreased risk of new vertebral fractures compared with those receiving placebo [Greenspan et al. 2007]. However, the magnitude of the reduction was changed with assumptions about fracture incidence in patients who failed to complete the study because only $67.2 \%$ of women who received at least one dose of the study drug completed the trial. Side effects including hypercalciuria, hypercalcemia and nausea were more 
common in the PTH group compared with those receiving a placebo drug.

Serum calcium should be assessed at 1, 3 and 6 months after initiation of treatment from a safety perspective.

\section{Teriparatide: safety issues}

Therapy with teriparatide is limited to 2 years because of the development of osteogenic sarcomas in rat studies. This appeared to be related to dose, duration and timing of therapy (young immature skeleton versus adult skeleton). There are currently no data to suggest that there is an increased risk of osteosarcoma in humans receiving teriparatide. Nevertheless, teriparatide is contraindicated in patients with 'Paget disease of bone or unexplained elevations of alkaline phosphatase, open epiphyses, or prior radiation therapy involving skeleton, children or growing adults, patients with bone metastases or history of skeletal malignancies and those with metabolic bone diseases other than osteoporosis' (Forsteo summary of product characteristics, Eli Lilly, http://www.medicines.ie/medicine/6405/SPC/

Forsteo/).

\section{Strontium ranelate: efficacy}

Strontium ranelate is a dual action bone agent. Its mechanism of action is unclear but it potentiates osteoblast proliferation and differentiation along with inhibiting osteoclast activity at resorption sites.

It is approved for the treatment of postmenopausal osteoporosis and is given in doses of $2 \mathrm{~g}$ daily. Ideally, it should be ingested at least $2 \mathrm{~h}$ after food, milk or calcium tablets. Strontium is chemically similar to calcium and preferentially binds to sites of trabecular rich bone. A recently published RCT looked at whether strontium ranelate reduced the risk of vertebral and nonvertebral fractures over a 5-year period [Seeman et al. 2010]. A subgroup of 1489 female patients over 80 years of age (mean $83.5 \pm 3.0$ years) with osteoporosis from the Spinal Osteoporosis Therapeutic Intervention (SOTI) and Treatment of Peripheral Osteoporosis (TROPOS) studies were randomized to receive either strontium ranelate $2 \mathrm{~g} /$ day or placebo and all participants received a combination of calcium and vitamin D. The results showed significant decreases in vertebral fracture risk (31\%), nonvertebral fracture $(27 \%)$ and major nonvertebral fracture risk $(33 \%)$.

\section{Strontium ranelate: safety issues}

Strontium is contraindicated in patients with a GFR less than $30 \mathrm{ml} / \mathrm{min}$ and coadministration with oral tetracyclines, quinolones and magnesium-containing antacids is best avoided as these compounds decrease the absorption of strontium. The most common side effects alluded to in clinical trials were nausea and diarrhoea over 5 years of treatment. Patients on longer courses of strontium reported memory loss and diarrhoea [Deeks and Dhillon, 2010].

In 2008, the European Medicine Agency (EMEA) issued an alert on the use of strontium ranelate and the risk of DRESS syndrome [EMEA, 2007]. This is a rare hypersensitivity reaction, typified by a triad of fever, rash and eosinophilia, with systemic symptoms including hepatitis and nephritis [Pernicova et al. 2008].

In a pooled analysis of clinical trials, strontium was positively correlated with an increased risk of venous thromboembolism [O'Donnell et al. 2006]. The findings indicated that the risk of venous thromboembolism and pulmonary embolism is slightly increased when taking a daily dosage of $2 \mathrm{~g}$ of strontium ranelate over a 3- to 4 -year period. The reason for this increased risk of thromboembolic disease is not clearly understood. In light of these findings the EMEA recommended close monitoring of this medication [EMEA, 2004]. The authors find that strontium is generally tolerated well, particularly in patients who find bisphosphonates difficult to tolerate.

\section{Hormone replacement therapy: efficacy}

Oestrogen provides a protective effect on the skeleton, which is illustrated by the rapid bone loss which follows the onset of menopause in women. Hormone replacement therapy (HRT) was the first choice treatment for postmenopausal osteoporosis prior to the introduction of bisphosphonates in the early $1990 \mathrm{~s}$. A number of different oestrogen and progestogen compounds are available for the treatment of osteoporosis. Oestrogens are available in oral form for both oestrogen-only regimens and combination therapy of oestrogen and progesterone. Oestrogen is also available as a transdermal patch. A combination of oestrogen and progestogen therapy is recommended in women with an intact uterus. Dosage is often varied cyclically, with oestrogens taken on a daily basis and progestogens taken for about 2 weeks every month, which is known as sequentially combined HRT. An alternative 
dosing regimen which involves both types of hormones being taken daily is known as continuous combined HRT. Progestogens such as norethisterone or medroxyprogestorone acetate are available in oral forms and levonergestrel is available as a transdermal patch. HRT has beneficial effects with regards to reduction of fracture risk as proven in the Women's Health Initiative (WHI) oestrogen-alone study [Andersen et al. 2004]. This was a large RCT which aimed to evaluate the effects of the most popularly prescribed postmenopausal hormone therapy medications in the United States on the incidence of chronic disease in a heterogenous population of healthy postmenopausal women. The results showed that conjugated equine oestrogen had a positive effect on fracture risk reduction in the areas of hip, clinical vertebral, and other fractures. Observed risks and benefits appeared equivalent over an average follow-up term of 6-8 years. These results concurred with evidence from prior observational studies [Weiss et al. 1980] and meta-analyses [Wells et al. 2002].

The WHI oestrogen plus progestin trial, which utilized a fixed combination of conjugated equine estrogens $0.625 \mathrm{mg}$ and medroxyprogesterone acetate $2.5 \mathrm{mg}$, endorsed the previous findings by Wells and colleagues, and confirmed a HRT-mediated reduction in hip and spine fractures [Cauley et al. 2003]. This trial established that oestrogen plus progestin resulted in increases in BMD and reduced fracture risk in healthy postmenopausal women. Baseline characteristics of women who benefited from the decreased fracture risk attributed to oestrogen plus progestin were homogenous in terms of age, body mass index, smoking history, history of falls, family history of fracture, total calcium intake, previous HRT use, BMD, or summary fracture risk score. The use of HRT for the primary prevention of osteoporosis in women with increased risk of fractures has not, as yet, been the basis of any large RCT.

\section{HRT: safety issues}

The aforementioned study [Andersen et al. 2004] showed that there was a significant correlation between HRT therapy and an increased risk of stroke, representing an excess risk of 12 cases per 10000 person-years in women with previous hysterectomy over an average of 6.8 years. In women who had not had hysterectomies, the WHI trial suggested that a combination of oestrogen and progestin therapy increased the risk of incident breast cancer [Roussouw et al. 2002].

A positive correlation between increased venous thromboembolism risk and postmenopausal oestrogen therapy has also been established, with the highest risk being attributed to the first year of use [Nelson et al. 2002].

As a result of these adverse findings, the authors find that the use of HRT in the treatment of postmenopausal osteoporosis has a limited role and necessitates careful weighing up of relative risks and benefits when evaluating patients for possible treatment.

\section{Selective oestrogen receptor modulators: efficacy}

Selective oestrogen receptor modulators (SERMs) act as weak oestrogens, and were initially launched as a safer replacement for HRT in the management of postmenopausal osteoporosis. However, the role of SERMs in the clinical treatment of postmenopausal osteoporosis is currently viewed as limited.

\section{Raloxifene}

Raloxifene is the only SERM approved for the prevention and treatment of postmenopausal osteoporosis and the recommended daily dose is $60 \mathrm{mg}$ daily.

The Multiple Outcomes of Raloxifene Evaluation (MORE) trial, which evaluated raloxifene as a treatment for postmenopausal osteoporosis, showed that dosages of both 60 and $120 \mathrm{mg}$ daily had a positive effect on vertebral fracture risk reduction [Ettinger et al. 1999]. Over a 4year study period, the lower dose of raloxifene $(60 \mathrm{mg} /$ day) showed a relative risk (RR) for one or more new vertebral fractures of $0.64[95 \%$ confidence interval (CI) 0.53 to 0.76 ] and a RR of 0.57 (95\% CI 0.48 to 0.69 ) for the higher dose of raloxifene $(120 \mathrm{mg} /$ day) [Delmas et al. 2002]. No significant effect on nonvertebral fracture was seen. A recently published meta-analysis stated that raloxifene (60 and $120 \mathrm{mg}$ doses combined) resulted in significant risk reductions in all clinical fractures and morphometric fractures in women [Kanis et al. 2010].

\section{Safety issues with SERMs}

Despite the evidence that SERMs such as raloxifene exert beneficial effects on the skeleton in an oestrogen-deficient state, use of these agents is 
limited by a small but significant risk of adverse outcomes, such as increased risk of stroke or thromboembolism [Ettinger et al. 1999].

SERMs are contraindicated in premenopausal women or those breastfeeding and are not advised for use with concomitant oestrogen replacement therapy. They should be discontinued $72 \mathrm{~h}$ before prolonged confinement or surgery with a high risk of thromboembolism. SERMs should also be avoided in patients with hepatic or renal dysfunction.

New SERMs, including bazedoxifene [Silverman et al. 2009] and lasofoxifene [Cummings et al. 2008], which have a higher anabolic effect on bone and reduced uterine side effects, are currently under development.

\section{RANK ligand inhibitors: efficacy}

\section{Denosumab}

Denosumab was first approved by the FDA in June 2010. It acts as a receptor activator of RANKL, thereby arresting osteoclast activity, decreasing bone resorption, resulting in gains in BMD. A large RCT randomized 7868 women with osteoporosis (age range 60-90 years) to receive either denosumab $60 \mathrm{mg}$ subcutaneously every 6 months for 36 months or placebo. The results showed a decreased risk of new radiographic vertebral fractures in women with a cumulative incidence of $2.3 \%$ in the denusomab group versus $7.2 \%$ in the placebo group, which is a relative decrease of $68 \%$ over 3 years [Cummings et al. 2009].

Denusomab is approved for use in postmenopausal women with osteoporosis and high fracture risk (i.e. a history of osteoporotic fracture, failed other treatments) and is licensed as a $60 \mathrm{mg}$ subcutaneous injection every 6 months.

\section{RANK ligand inhibitors: safety issues}

Hypocalcemia must be corrected before initiating treatment and caution must be observed in patients with a decreased GFR less than $30 \mathrm{ml} /$ min or patients on haemodialysis.

Concerning features of preclinical trials include the fact that no carcinogenicity studies were performed due to a lack of an animal model. Three patients in a high-dose denosumab group in the dose-finding trial died of a new malignancy [FDA, 2009].
In the primary postmenopausal osteoporosis studies, there was an imbalance in the incidence of malignancies in the denosumab group, driven by breast, reproductive and gastrointestinal cancers.

Further concerns with regards to increased risk of infection centres around the fact that denusomab is an inhibitor of RANKL, which plays a crucial role in B-cell and T-cell differentiation along with antigen-presenting cell survival. In preclinical trials, there was an imbalance of serious infections in the denosumab group, most notably skin, ear and urinary tract infections. There was also an imbalance in infective arthritis but no evident increase in opportunistic infections [FDA, 2009].

One case report of a patient with malignancy on denosumab who developed ONJ without previous history of irradiation or bisphosphonate administration has been published [Taylor et al. 2010]. This association appears to be dose related, which is a similar phenomenon to bisphosphonate-related ONJ.

Further postmarketing research is needed to evaluate these potentially serious adverse events.

\section{Summary}

All the above drug classes can reduce the risk of fracture in men and women with osteoporosis. When considering the various adverse events associated with the different classes, in particular bisphosphonates, it is important in each case to consider the strength of the evidence for a causal relationship, the risk-benefit profile of the chosen drug and the characteristics of each individual patient.

Although safety issues are of paramount importance, it is important that clinicians are not deterred from recognizing the value that appropriate use of these drugs can achieve in reducing fractures in many patients with osteoporosis.

Further research is needed, particularly on the newer agents, in order to establish postmarketing safety and expand their safety profile.

\section{Funding}

This research received no specific grant from any funding agency in the public, commercial, or notfor-profit sectors. 


\section{Conflict of interest statement}

The authors declare no conflicts of interest in preparing this article.

\section{References}

Abrahamsen, B., Eiken, P. and Eastell, R. (2009) Subtrochanteric and diaphyseal femur fractures in patients treated with alendronate: A register-based national cohort study. $\mathcal{F}$ Bone Miner Res

24: 1095-1102.

Ahmed, S.F. and Elmantaser, M. (2009) Secondary osteoporosis. Endocr Dev 16: 170-190.

Anderson, G.L., Limacher, M., Assaf, A.R., Bassford, T., Beresford, S.A., Black, H. et al. (2004) Effects of conjugated equine estrogen in postmenopausal women with hysterectomy: The Women's Health Initiative Randomised Controlled Trial. FAMA

291: 1701-1712.

Andersen, M. and Kukreja, S. (2008)

Bisphosphonates and chronic kidney disease. Kidney 17: 119-121.

Bischoff-Ferrari, H.A., Conzelmann, M., Stähelin, H.B., Dick, W., Carpenter, M.G., Adkin, A.L. et al. (2006) Is fall prevention by vitamin D mediated by a change in postural or dynamic balance? Osteoporos Int 17: 656-663.

Bischoff-Ferrari, H.A., Willett, W.C., Wong, J.B., Stuck, A.E., Staehelin, H.B., Orav, E.J. et al. (2009) Prevention of nonvertebral fractures with oral vitamin $\mathrm{D}$ and dose dependency: A meta-analysis of randomized controlled trials. Arch Intern Med 169: 551-561.

Black, D.M., Cummings, S.R., Karpf, D.B., Cauley, J.A., Thompson, D.E., Nevitt, M.C. et al. (1996) Randomised trial of effect of alendronate on risk of fracture in women with existing vertebral fractures. Fracture Intervention Trial Research Group. Lancet 348: 1535-1541.

Black, D.M., Delmas, P.D., Eastell, R., Reid, I.R., Boonen, S., Cauley, J.A. et al. (2007) HORIZON Pivotal Fracture Trial. Once-yearly zoledronic acid for treatment of postmenopausal osteoporosis. $N$ Engl $\mathcal{F}$ Med 356: 1809-1822.

Blair, H.C., Teitelbaum, S.L., Ghiselli, R. and Gluck, S. (1989) Osteoclastic bone resorption by a polarized proton pump. Science 245: 855-857.

Bolland, M.J., Avenell, A., Baron, J.A., Grey, A., MacLennan, G.S., Gamble, G.D. et al. (2010) Effect of calcium supplements on risk of myocardial infarction and cardiovascular events: meta-analysis. BMF 341: c3691.

Bolland, M.J., Hay, D., Grey, A., Reid, I.R. and Cundy, T. (2006) Osteonecrosis of the jaw and bisphosphonates - putting the risk in perspective. $N Z$ Med F 118: U2339.

Boonen, S., Sellmeyer, D.E., Lippuner, K., OrlovMorozov, A., Abrams, K., Mesenbrink, P. et al. (2008) Renal safety of annual zoledronic acid infusions in osteoporotic postmenopausal women. Kidney Int 74: $641-648$.

Burge, R.T., Worley, D., Johansen, A., Bhattacharyya, S. and Bose, U. (2001) The cost of osteoporotic fractures in the UK: Projections for 2000-2020. F Med Econ 4: 51-62.

Cardwell, C.R., Abnet, C.C., Cantwell, M.M. and Murray, L.J. (2010) Exposure to oral bisphosphonates and risk of oesophageal cancer. FAMA 304: 657-663.

Cauley, J.A., Robbins, J., Chen, Z., Cummings, S.R., Jackson, R.D., LaCroix, A.Z. et al. (2003) Effects of estrogen plus progestin on risk of fracture and bone mineral density. The Women's Health Initiative randomized trial. $\mathcal{F} A M A$ 290: 1729-1738-.

Chaiamnuay, S. and Saag, K.G. (2006)

Postmenopausal osteoporosis What have we learned since the introduction of bisphosphonates? Rev Endocr Metab Disord 7: 101-112.

Chesnut, I.C., Skag, A., Christiansen, C., Recker, R., Stakkestad, J.A., Hoiseth, A. et al. (2004) Effects of oral ibandronate administered daily or intermittently on fracture risk in postmenopausal osteoporosis. $\mathcal{F}$ Bone Miner Res 19: 1241-1249.

CHMP (2009) Assessment report on bisphosphonates and osteonecrosis of the jaw. Available at: http:// www.emea.europa.eu (accessed 8 May 2011).

Cleland, J.G.F., Witte, K. and Steel, S. (2010) Calcium supplements in people with osteoporosis. BMf 341: c3856.

Cranney, A., Tugwell, P., Adachi, J., Weaver, B., Zytaruk, N., Papaioannou, A. et al. (2002) Meta-analyses of therapies for postmenopausal osteoporosis. III. Meta-analysis of risedronate for the treatment of postmenopausal osteoporosis. Endocr Rev 23: $517-523$.

Cummings, S.R., Black, D.M., Thompson, D.E., Applegate, W.B., Barrett-Connor, E., Musliner, T. et al. (1998) Effect of alendronate on risk of fracture in women with low bone density but without vertebral fractures: Results from the Fracture Intervention Trial. FAMA 280: 2077-2082.

Cummings, S.R., Eastell, R., Ensrud, K., Reid, D.M., Vukicevic, S., Lacroix, A. et al. (2008) The effects of lasofoxifene on fractures and breast cancer: 3-year results from the PEARL trial. F Bone Miner Res 23(Suppl. 1): S81.

Cummings, S.R., San Martin, J., McClung, M.R., Siris, E.S., Eastell, R., Reid, I.R. et al. (2009) Denosumab for prevention of fractures in postmenopausal women with osteoporosis. N Engl f Med 391: 756-765.

Cunningham, J., Sprague, S.M., Cannata-Andia, J., Coco, M., Cohen-Solal, M., Fitzpatrick, L. et al. Osteoporosis Work Group. (2004) Osteoporosis in chronic kidney disease. Am F Kidney Dis 43: 566-571.

Dawson-Hughes, B. (2010) Reply to 'Effect of calcium supplements on risk of myocardial infarction and 
cardiovascular events: meta-analysis'. BMF 341: c3691. Available at: http://www.bmj.com/content/341/ bmj.c3691/reply (accessed 8 May 2011).

Dawson-Hughes, B., Mithal, A., Bonjour, J.P., Boonen, S., Burckhardt, P., Fuleihan, G.E. et al. (2010) IOF position statement: Vitamin D recommendations for older adults. Osteoporos Int 21: 1151-1154.

Deeks, E.D. and Dhillon, S. (2010) Spotlight on strontium ranelate: In postmenopausal osteoporosis. Drugs Aging 27: 771-773.

Delmas, P.D., Ensrud, K.E., Adachi, J.D., Harper, K.D., Sarkar, S., Gennari, C. et al. (2002) Efficacy of raloxifene on vertebral fracture risk reduction in postmenopausal women with osteoporosis:

Four-year results from a randomized clinical trial. f Clin Endocrinol Metab 87: 3609-3617.

Delmas, P.D., Recker, R.R., Chesnut, E.C.H., Skag, A., Stakkestad, J.A., Emkey, E.R. et al. (2004) Daily and intermittent oral ibandronate normalize bone turnover and provide significant reduction in vertebral fracture risk: Results from the BONE study. Osteoporos Int 15: 792-798.

DIPART (Vitamin D Individual Patient Analysis of Randomized Trials) Group. (2010) Patient level pooled analysis of 68500 patients from seven major vitamin D fracture trials in US and Europe. BMF 340: b5463.

EMEA (2004) 2004-Protelos. The Committee for Medicinal Products for Human Use.

EMEA (2007) 2007-Protelos. Press release. Available at: http://www.emea.europa.eu/humandocs/PDFs/ EPAR/protelos/PressRelease_Protelos 41745807en.pdf (accessed 8 May 2011).

Eriksen, E.F., Langdahl, B., Vesterby, A., Rungby, J. and Kassem, M. (1999) Hormone replacement therapy prevents osteoclastic hyperactivity: A histomorphometric study in early postmenopausal women. $\mathcal{F}$ Bone Miner Res 14: 1217-1221.

Ettinger, B., Black, D.M., Mitlakm, B.H., Knickerbocker, R.K., Nickelsen, T., Genant, H.K. et al. (1999) Reduction of vertebral fracture risk in postmenopausal women with osteoporosis treated with raloxifene: Results from a 3-year randomized clinical trial. Multiple Outcomes of Raloxifene Evaluation (MORE) Investigators. $\mathcal{F} A M A$ 282: 637-645.

FDA (2008) FDA update 2008. Available at: http:// www.fda.gov./cder/drug/early_comm/ bisphosphonates_update_200811.htm (accessed 8 May 2011).

FDA (2009) Denosumab meeting briefing document, 13 August. FDA Update Advisory Committee for Reproductive Health Drugs. Available at: http:// www.fda.gov/downloads/AdvisoryCommittees/ CommitteesMeetingMaterials/Drugs/ ReproductiveHealthDrugsAdvisoryCommittee/ UCM176623.pdf (accessed 15 December 2010).
Fraunfelder, F.W. and Fraunfelder, F.T. (2003) Bisphosphonates and ocular inflammation. $N$ Engl $\mathcal{F}$ Med 348: 1187-1188.

Fraunfelder, F.W., Fraunfelder, F.T. and Jensvold, B. (2003) Scleritis and other ocular side effects associated with pamidronate disodium. Am $\mathcal{F}$ Ophthalmol 135: 219-222.

Green, J., Czanner, G., Reeves, G., Watson, J., Wise, L. and Beral, V. (2010) Oral bisphosphonates and risk of cancer of oesophagus, stomach, and colorectum: Case-control analysis within a UK primary care cohort. BMF 341: c4444.

Greenspan, S.L., Bone, H.G., Ettinger, M.P., Hanley, D.A., Lindsay, R., Zanchetta, J.R. et al. (2007) Effect of recombinant human parathyroid hormone (1-84) on vertebral fracture and bone mineral density in postmenopausal women with osteoporosis: A randomized trial. Ann Intern Med 146: 326-339.

Hughes, D.E., Dai, A., Tiffee, J.C., Li, H.H., Mundy, G.R., Boyce, B.F. et al. (1996) ER promotes apoptosis of murine osteoclasts mediated by TGF-beta. Nat Med 2: 1132-1136.

Jamal, S.A., Bauer, D.C., Ensrud, K.E., Cauley, J.A., Hochberg, M., Ishani, A. et al. (2007) Alendronate treatment in women with normal to severely impaired renal function: An analysis of the fracture intervention trial. J Bone Miner Res 22: 503-508.

Kanis, J.A., Johansson, H., Oden, A. and McCloskey, E.V. (2010) A meta-analysis of the efficacy of raloxifene on all clinical and vertebral fractures and its dependency on FRAX(R). Bone 47: 729-735.

Keen, R. (2007) Osteoporosis: Strategies for prevention and management. Best Pract Res Clin Rheumatol 21: $109-122$.

Klotzbuecher, C., Ross, P., Landsman, P., Abbott, T. and Berger, M. (2000) Patients with prior fractures have an increased risk of future fractures: A summary of the literature and statistical synthesis. F Bone Miner Res 15: 721-739.

Kroll, M.H. (2000) Parathyroid hormone temporal effects on bone formation and resorption. Bul Math Biol 62: 163-187.

Lenart, B.A., Lorich, D.G. and Lane, J.M. (2008) Atypical fractures of the femoral diaphysis in postmenopausal women taking alendronate. $N$ Engl F Med 58: $1304-1306$.

Lyles, K.W., Colón-Emeric, C.S., Magaziner, J.S., Adachi, J.D., Pieper, C.F., Mautalen, C. et al. (2007) Zoledronic acid and clinical fractures and mortality after hip fracture. N Engl f Med 357: 1799-1809.

Macarol, V. and Fraunfelder, F.T. (1994) Pamidronate disodium and possible ocular adverse drug reactions. Am $\mathcal{F}$ Ophthalmol 188: 220-224.

Markowitz, G.S., Fine, P.L., Stack, J.I., Kunis, C.L., Radhakrishnan, J., Palecki, W. et al. (2003) Toxic acute tubular necrosis following treatment with zoledronate. Kidney Int 64: 281-289. 
MHRA (2009) MHRA drug safety update, March 2009. Medicines and Healthcare Products Regulatory Agency. Available at: http://www.mhra.gov.uk/ Publications/Safetyguidance/DrugSafetyUpdate/ CON041211.

Miller, P.D., Roux, C., Boonen, S., Barton, I.P., Dunlap, L.E. and Burgio, D.E. (2005) Safety and efficacy of risedronate in patients with age-related reduced renal function as estimated by the Cockcroft and Gault method: A pooled analysis of nine clinical trials. $\mathcal{F}$ Bone Miner Res 20: 2105-2215.

National Institute for Health and Clinical Excellence (2008) Alendronate, etidronate, risedronate, raloxifene, strontium and teriparatide for the secondary prevention of osteoporotic fragility fractures in postmenopausal women, October. Available at: www.nice.org.uk (accessed 8 May 2011).

National Osteoporosis Foundation (NOF). (2010) Clinician's Guide to Prevention and Treatment of Osteoporosis, Washington, DC: National Osteoporosis Foundation.

Nelson, H.D., Humphrey, L.L., Nygren, P., Teutsch, S.M. and Allan, J.D. (2002) Postmenopausal hormone replacement therapy and risk for venous thromboembolism. FAMA 288: 872-881.

O’Donnell, S., Cranney, A., Wells, G.A., Adachi, J.D. and Reginster, J.Y. (2006) Strontium ranelate for preventing and treating postmenopausal osteoporosis. Cochr Database Syst Rev (3): CD005326.

Odvina, C.V., Zerwekh, J.E., Rao, D.S., Maalouf, N., Gottschalk, F.A. and Park, C.Y. (2005) Severely suppressed bone turnover: A potential complication of alendronate therapy. $\mathcal{F}$ Clin Endocrinol Metab 90: 1294-1301.

Perazella, M.A. and Markowitz, G.S. (2008) Bisphosphonate nephrotoxicity. Kidney Int 74: 1385-1393.

Pernicova, I., Midleton, E.T. and Aye, M. (2008) Rash, strontium ranelate and DRESS syndrome put into perspective. European Medicine Agency on the alert. Osteoporos Int 19: 1811-1812.

Peter, R., Mishra, V. and Fraser, W.D. (2004) Severe hypocalcemia after being given intravenous bisphosphonate. BMF 328: 335-336.

Pols, H.A., Felsenberg, D., Hanley, D.A., Stepan, J., Munoz-Torres, M., Wilkin, T.J. et al. (1999) Multinational, placebo controlled, randomized trial of the effects of alendronate on bone density and fracture risk in postmenopausal women with low bone mass: Results of the FOSIT study. Fosamax International Trial Study Group. Osteoporos Int 9: 461-468.

Reid, I.R., Ames, R., Mason, B., Bolland, M.J., Bacon, C.J., Reid, H.E. et al. (2010) Effects of calcium supplementation on lipids, blood pressure and body composition in older men: A randomised controlled trial. Am f Clin Nutr 91: 131-139.
Reid, I.R., Brown, J.P., Burckhardt, P., Horowitz, Z., Richardson, P., Trechsel, U. et al. (2002) Intravenous zoledronic acid in postmenopausal women with low bone mineral density. $N$ Engl $\mathcal{F}$ Med 346: 653-661.

Rosen, C.J., Hochberg, M.C., Bonnick, S.L., McClung, M., Miller, P., Broy, S. et al. (2005)

Treatment with once weekly alendronate $70 \mathrm{mg}$ compared with once-weekly risedronate $35 \mathrm{mg}$ in women with postmenopausal osteoporosis: A randomized double-blind study. F Bone Miner Res 20: 141-151.

Roussouw, J.E., Anderson, G.L., Prentice, R.L., LaCroix, A.Z., Kooperberg, C., Stefanick, M.L. et al. (2002) Risks and benefits of estrogen plus progestin in healthy postmenopausal women. $\mathcal{F} A M A$ 288: 321-333.

Rustemeyer, J. and Bremerich, A. (2010)

Bisphosphonate-associated osteonecrosis of the jaw: What do we currently know? A survey of knowledge given in the recent literature. Clin Oral Investig 14: 59-64.

Sanders, K.M., Stuart, A.L., Williamson, E.J., Simpson, J.A., Kotowicz, M.A., Young, D. et al. (2010) Annual high-dose oral vitamin D and falls and fractures in older women: a randomized controlled trial. $\mathcal{F} A M A$ 303: 1815-1822.

Schmidt, G.A., Horner, K.E., McDanel, D.L., Ross, M.B. and Moores, K.G. (2010) Risks and benefits of long-term bisphosphonate therapy. Am $\mathcal{F}$ Health Syst Pharm 67: 994-1001.

Seeman, E., Boonen, S., Borgström, F., Vellas, B., Aquino, J.P., Semler, J. et al. (2010) Five years treatment with strontium ranelate reduces vertebral and nonvertebral fractures and increases the number and quality of remaining life-years in women over 80 years of age. Bone 46: 1038-1042.

Seeman, E. and Delmas, P.D. (2006) Bone quality - the material and structural basis of bone strength and fragility. N Engl f Med 354: 2250-2261.

Shane, E., Burr, D., Ebeling, P.R., Abrahamsen, B., Adler, R.A., Brown, T.D. et al. (2010) Atypical subtrochanteric and diaphyseal femoral fractures: Report of a task force of the American Society for Bone and Mineral Research. F Bone Miner Res 25: 2267-2294.

Silverman, S.L., Christiansen, C., Genant, H.K., Vukicevic, S., Zanchetta, J.R., de Villiers, T.J. et al. (2009) Efficacy of bazedoxifene in reducing new vertebral fracture risk in postmenopausal women with osteoporosis: Results from a 3-year, randomized, placebo, and active-controlled clinical trial. F Bone Miner Res 23: 1923-1934.

Tang, B.M., Eslick, G.D., Nowson, C., Smith, C. and Bensoussan, A. (2007) Use of calcium or calcium in combination with vitamin $\mathrm{D}$ supplementation to prevent fractures and bone loss in people aged 50 years and older: A meta-analysis. Lancet 370: 657-666.

Taylor, K.H., Middlefell, L.S. and Mizen, K.D. (2010) Osteonecrosis of the jaws induced by anti-RANK 
ligand therapy. Br f Oral Maxillofac Surg 15, October [Epub ahead of print].

Trivedi, D.P., Doll, R. and Khaw, K. (2003)

Effect of four monthly oral vitamin D supplementation on fractures and mortality in men and women living in the community: Randomised double blind controlled trial. $B M F$ 326: 469-475.

Watts, N.B. and Diab, D.L. (2010) Long term use of bisphosphonate in osteoporosis. F Clin Endocrinol Metab 97: 1555-1565.

Weiss, N.S., Ure, C.L., Ballard, J.H., Williams, A.R. and Daling, J.R. (1980) Decreased risk of fractures of the hip and lower forearm with postmenopausal use of estrogen. N Engl f Med 303: 1195-1198.

Wells, G., Tugwell, P., Shea, B., Guyatt, G., Peterson, J., Zytaruk, N. et al. (2002) Meta-analysis of the efficacy of hormone replacement therapy in treating and preventing osteoporosis in postmenopausal women. Endocrinol Rev 23: 529-539.
Woos, S., Hellstein, J. and Kalmar, J. (2006) Systematic review: Bisphosphonates and osteonecrosis of the jaws. Ann Internal Med 144: 753-761.

World Health Organization (2006) Fracture risk assessment tool. Available at http://www.shef.ac.uk/ FRAX/ (accessed 25 August 2010).

Wysowski, D.W. (2009) Reports of oesophageal cancer with oral bisphosphonate use. $N$ Engl FMed 360: 89-90.

Wysowski, D.W. (2010) Oral bisphosphonates and oeseophageal cancer. BMF 341: c4506.

Yasuda, H., Shima, N., Nakagawa, N., Yamaguchi, K., Kinosaki, M., Mochizuki, S. et al. (1998) Osteoclast differentiation factor is a ligand for osteoprotegerin/ osteoclastogenesis-inhibitory factor and is identical to TRANCE/RANKL. Proc Natl Acad Sci U S A 95: 3597-3602.

Zallone, A. (2006) Direct and indirect oestrogen actions on osteoblasts and osteoclasts. Ann N Y Acad Sci 1068: 173-179. 